\title{
Le cancer de la prostate : Acquis et challenges pour l'an 2000
}

\author{
M. Colombel, F. Blanc, J.M. Marechal
}

Service d'Urologie et Chirurgie de la Transplantation, Hôpital E. Herriot, Lyon 69003

\section{RESUME}

Quel que soit le stade et le degré d'évolution du cancer de la prostate, les principes des traitements, leur indications et les modalités de la surveillance sont en constante évolution. Le diagnostic précoce est accessible au clinicien qui dispose du PSA et du toucher rectal pour poser les indications des biopsies. Ceci permet aujourd'hui de traiter des patients à un stade précoce avec un meilleur pronostic lorsque le cancer est localisé à la glande. Les améliorations de la technique chirurgicale ont largement contribué à diminuer la morbidité postopératoire et celle-ci représente le traitement de référence. La radiothérapie reste une alternative au traitement chirurgical mais les résultats sont plus difficiles à analyser. Le traitement hormonal par déprivation androgénique est indiqué au stade des métastases. Les agonistes de la LHRH associés aux anti-androgènes représentent une alternative tout aussi efficace que la castration chirurgicale. Malheureusement, le pronostic des formes avancées reste sombre et imprévisible. Les objectifs pour les années à venir seront d'améliorer l'évaluation diagnostic du cancer de prostate et de mieux définir les indications en fonction des traitements disponibles; de mieux comprendre les effets du traitement hormonal et de proposer de nouveaux traitements pour les formes androgèno-indépendantes.

Mots Clés : Cancer de Prostate; Diagnostic ; Traitement : Physiopathologie

\section{INTRODUCTION}

Il est admis que le cancer de la prostate est le cancer le plus fréquent chez l'homme et qu'il représente la deuxième cause de mortalité par cancer. Son incidence va très probablement augmenter dans les prochaines années du fait $\mathrm{du}$ vieillissement de la population et du retentissement $d u$ " baby boom " des années quarante sur la pyramide des âges [24]. Au cours de la dernière décade de nombreux travaux de recherche clinique et fondamentale ont été réalisés, qui nous permettent aujourd'hui une meilleure approche thérapeutique en fonction de l'histoire naturelle de la maladie. Ces travaux sont également à la base de nouveaux traitements du cancer prostatique dont l'évaluation devrait voir le jour dans la prochaine décade. Pour le clinicien il est important de connaître les différents aspects du diagnostic et des traitements de cette maladie, les progrès réalisés, et les thèmes de recherche qui sont en cours de développement.

Correspondance : $\mathrm{Dr}$ Marc COLOMBEL, Service d'Urologie et Chirurgie de la Transplantation, Hôpital E. Herriot, 5 place d'Arsonval, LYON, 69437

Remerciements à la Ligue Contre le Cancer du Rhône qui nous permet de poursuivre nos travaux cliniques et scientifiques sur le sujet 


\section{CIRCONSTANCES DE DIAGNOSTIC ET PRISE EN CHARGE}

Le diagnostic précoce de cancer prostatique est possible par l'utilisation du dosage du PSA (valeur seuil de 2 à $4 \mathrm{ng} / \mathrm{ml}$ selon l'âge) et du toucher rectal. Pour certains, le dépistage est systématique dès l'âge de cinquante ans, il fait d'ailleurs partie du bilan de santé offert par la sécurité sociale. Le diagnostic histologique est indispensable, il peut être fait par biopsies ou à partir de l'analyse de copeaux de résection de prostate.

Aujourd'hui, c'est le plus souvent devant une élévation des PSA ( $>4 \mathrm{ng} / \mathrm{ml}$ ) que l'on est amené à réaliser des biopsies prostatiques. Celles ci sont réalisées selon une technique standardisée, en quadrant et sous contrôle echographique [32].

L'interprétation des biopsies demande une certaine expertise pour identifier des glandes tumorales et déterminer le score de Gleason, élément pronostic majeur et qui est la somme des grades histologiques ( 1 à 5 ) les plus fréquents ( Figure 1). Cependant, dans $20 \%$ des cas, le PSA est normal. C'est pourquoi, le toucher rectal reste un élément fondamental du diagnostic. Lorsqu'il existe une lésion palpable, ou une impression d'induration, les biopsies sont réalisées selon la même technique et complétées dans ce cas de biopsies dirigées sur la zone tumorale.

Toucher rectal et dosage des PSA sont donc les deux seuls éléments nécessaires au diagnostic du cancer prostatique [6]. L'échographie n'est pas un instrument de dépistage du cancer de prostate. Cet examen peut être réalisé en complément du PSA et du toucher rectal mais il ne permet pas d'améliorer les performances diagnostiques du toucher rectal et du dosage du PSA.

L'attitude systématique de diagnostic utilisant principalement le PSA est certainement à l'origine de l'augmentation du nombre de cas de cancer de prostate, de la baisse du pourcentage de patients diagnostiqués au stade des métastases et de la précocité du diagnostic avec des tumeurs de plus petit volume et plus fréquemment localisées à l'organe [7-28]. Néanmoins, il persiste un pourcentage impor- tant de patients qui ont un cancer de prostate à PSA normal et à l'inverse, de nombreux patients auront des biopsies négatives malgré un PSA élevé entre 4 et $10 \mathrm{ng} / \mathrm{ml}$. Il faut également tenir compte des variations du PSA en fonction de l'âge qui modulent les indications de la biopsie (Tableau 1). C'est pourquoi d'autres tests du PSA ont été développés. Ces derniers reposent sur le principe de l'élévation anormale de PSA dans le cas du cancer prostatique et tiennent compte soit du volume prostatique total (densité du PSA< 0.15), de la vitesse d'augmentation du PSA (vélocité du $\mathrm{PSA}>0.5 \mathrm{ng} / \mathrm{an}$ ) soit plus récemment de la proportion de PSA libre dans le sang circulant (issu des glandes normales et dont le rapport avec le PSA total, conjugué doit être $>20 \%$ ) [23]. Ces tests permettent de mieux cerner les indications de la biopsie de prostate pour les patients qui présentent des troubles mictionnels, avec un toucher rectal normal et dont le PSA est compris entre 4 et $10 \mathrm{ng} / \mathrm{ml}$.

D'autres éléments cliniques sont essentiels pour la prise en charge de ces patients. Il s'agit de l'âge et de l'existence de co-morbidités qui déterminent l'espérance de vie [13]. En effet, en l'absence de symptômes, du fait d'un bénéfice de survie significatif entre 5 et 10 ans, le traitement radical n'est indiqué que pour les patients dont l'espérance de vie est supérieure en règle à 10 ans pour le traitement chirurgical. Les résultats des études cliniques, montrent effectivement que la surveillance simple est possible en cas de cancer bien différencié, non palpable et avec une espérance de vie de moins de 10 ans. Dans tous les autres cas, le traitement est justifié et également, le diagnostic à un stade précoce.

Tableau 1 Valeurs du PSA (ng/ml) en fonction de l'âge

\begin{tabular}{lll} 
Age & PSA $(\mathbf{n g} / \mathbf{m l})$ & $\begin{array}{l}\text { Densité du PSA } \\
(\mathbf{n g} / \mathbf{m l} / \mathbf{m l})\end{array}$ \\
\hline $40-49$ & $0.0-2.5$ & $0.0-0.08$ \\
$50-59$ & $0.0-3.5$ & $0.0-0.1$ \\
$60-69$ & $0.0-4.5$ & $0.0-0.11$ \\
$70-79$ & $0.0-6.5$ & $0.0-0.13$ \\
\hline
\end{tabular}




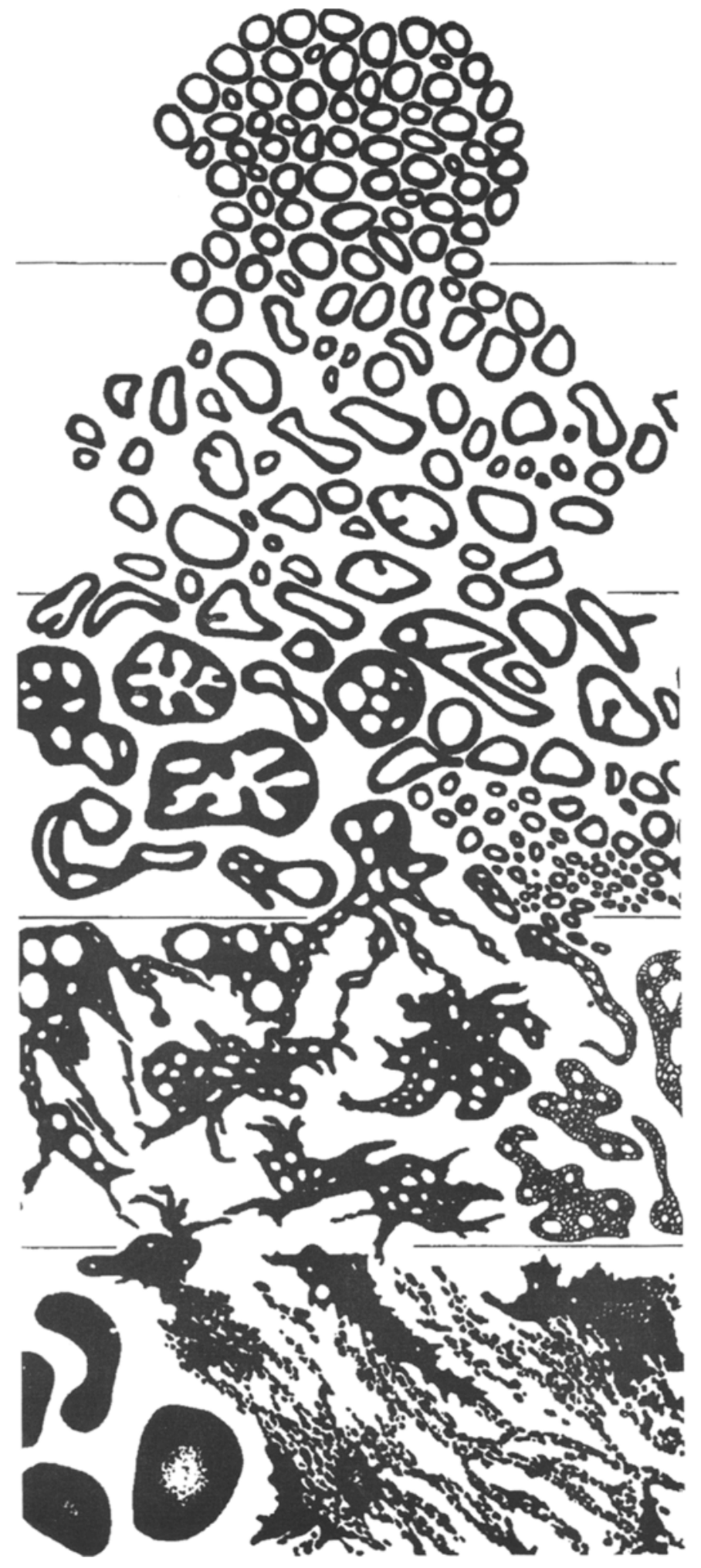
Grade 1 : petites glandes très bien différenciées, de taille régulière

Grade 2 : petites glandes bien différenciées, de taille irrégulière

Grade 3: petites glandes
moyennement différenciées, de taille
irrégulière

Grade 4: petites glandes peu différenciées, de taille irrégulière, confluantes

Grade 5: petites glandes très indifférenciées, cellules anaplasiques

Figure 1 A. Schéma représentant les différentes formes de différenciation du cancer de prostate, selon Gleason, B. Aggrandissement X40 d'un cancer de prostate moyennement différencié grade III de Gleason 
Lorsque le diagnostic est posé et qu'il s'agit d'un cancer asymptomatique, le choix des examens complémentaires est standardisé. Du fait du caractère lymphophile et surtout ostéophile de cette tumeur, les examens de références à la recherche de lésions secondaires sont le scanner abdomino-pelvien et la scintigraphie osseuse. Certains auteurs ont montré que le risque de métastases ganglionnaires est négligeable lorsque le taux de PSA est inférieur à $20 \mathrm{ng} / \mathrm{ml}$ [14]. Le risque d'avoir des métastases osseuses est également très faible mais il reste suffisamment important (de 4 à $7 \%$ ) pour que l'on propose de réaliser cet examen à titre systématique qui servira également de référence pour le suivi carcinologique [2]. D'autres examens devraient être développés dans un avenir proche, comme la scintigraphie au glucose, la reconnaissance des cellules tumorales circulantes, l'identification de marqueurs histo-pronostiques à partir des biopsies.

Avant toute décision thérapeutique et disposant du diagnostic histologique, du bilan lésionnel et métastatique, le clinicien doit donner un stade au cancer en s'aidant de la classification (Tableau 2) de l'OMS 1997 et lorsqu'il s'agit d'un stade localisé T1 ou T2, apprécier les risques d'une maladie déjà extensive pour laquelle le traitement chirurgical expose au risque de marges positives et donc à la récidive post opératoire. Nous disposons pour cela de plusieurs paramètres indépendants (PSA, Gleason, Toucher rectal) qui permettent d'apprécier ce risque avec une bonne fiabilité [25].

Dans le cas des cancer avancés au stade des métastases, ou de l'envahissement ganglion-

Tableau 2 : Classification OMS 1997 du cancer de prostate, ne s'applique qu'aux adénocarcinomes, les tumeurs urothéliales de la prostate doivent être classées comme les tumeurs de l'urethre

\section{Stade}

\begin{tabular}{l|l}
\hline Tx & La tumeur primitive ne peut être identifiée \\
\hline T0 & Aucune tumeur
\end{tabular}

\section{T1 : Tumeur cliniquement impalpable}

\begin{tabular}{l|l}
\hline T1a & Découverte fortuite sur moins de $5 \%$ des copeaux de résection \\
\hline T1b & Découverte fortuite sur plus de $5 \%$ des copeaux de résection \\
\hline T1c & Tumeur identifiée par biopsie (lors d'une élévation des PSA) \\
\hline
\end{tabular}

T2 : Tumeur cliniquement confinée à la prostate

\begin{tabular}{l|l}
\hline T2a & Envahissant un lobe \\
\hline $\mathrm{T} 2 \mathrm{~b}$ & Envahissant les deux lobes \\
\hline
\end{tabular}

T3 : Tumeur avec extension clinique extra prostatique

\begin{tabular}{l|l}
\hline T3a & Extension uni ou bi latérale \\
\hline T3b & Envahissant les vésicules séminales \\
\hline
\end{tabular}

T4 :Tumeur envahissant cliniquement les structures adjacentes 
naire, le traitement radical ne sera pas proposé. Seul le traitement par déprivation androgénique (castration chirurgicale ou médicale) permet d'obtenir une rémission et nous ne disposons dans ces circonstances que de peu d'outils thérapeutiques et pronostiques pour faire face à la progression des métastases qui s'avère inéluctable.

Il est donc essentiel avant le diagnostic de déterminer quels sont les patients qui doivent subir une biopsie de prostate, et après le diagnostic de déterminer quels sont les patients qui doivent être traités et par quelle méthode.

\section{DÉCISION DU TRAITEMENT RADICAL}

Le traitement chirurgical a considérablement évolué au cours de la dernière décade. Le contrôle des plexus veineux préprostatiques permet de réaliser une dissection précise et exsangue de l'apex prostatique. Dans notre expérience, les pertes moyennes sanguines sont inférieures à $500 \mathrm{ml}$, très peu de patients sont transfusés, l'incontinence postopératoire est devenue exceptionnelle. Le refoulement des pédicules caverneux par une dissection intrafasciale lorsqu'elle est possible (tumeur non palpable) permet aux patients les plus jeunes une meilleure récupération de leur fonction sexuelle [35]. Enfin, lorsque la tumeur est confinée à l'organe le taux de survie spécifique et de survie sans progression à très long terme est excellent et montre à quel point, la chirurgie est le seul moyen d'accéder à la guérison [17, 22, 26, 30].

Parallèlement au traitement chirurgical, les techniques de radiothérapies ont été améliorées, permettant de délivrer une énergie suffisamment importante sur un volume mieux défini et de limiter la toxicité rectale et vésicale [15]. Les résultats à long terme restent difficiles à interpréter. Les patients sont souvent plus âgés, et le stade pathologique de la maladie n'est pas connu, ce qui rend difficile de comparer les deux techniques. Cependant, beaucoup aujourd'hui considèrent que la radiothérapie n'est pas l'équivalent de l'exérèse tumorale, du fait de la persistance de glandes tumorales quelle que soit la technique utilisée, du risque de 2ème cancer prostatique [16], de la lenteur de la réponse au traitement (12 à 18 mois). Enfin, la radiothérapie présente des risques fonctionnels de douleurs périnéales chroniques, de sténoses de l'urèthre, de troubles mictionnels, de vessie et de rectite radique et ce traitement ne prévient pas le risque de dysérection. Les nouvelles techniques comme la radiothérapie conformationnelle ont été développées pour prévenir ces complications, et les résultats sur le plan fonctionnel sont en cours d'évaluation.

La radiothérapie reste cependant un outil thérapeutique important pour ralentir la progression tumorale des stades localement avancés et est aujourd'hui considérée comme complémentaire du traitement par déprivation androgénique. En effet, le traitement combiné hormonal et radiothérapie pour les tumeurs de stade T3 présente un avantage en terme de survie comparativement à la radiothérapie seule [5].

Lorsqu'il est possible, le traitement chirurgical est le seul moyen d'accéder à la guérison. Il faut reconnaître que malgré les progrès de la technique opératoire, le risque d'impuissance est majeur. Mais il s'agit de la seule séquelle, l'incontinence étant devenue rare de même que les complications chirurgicales. L'impuissance post opératoire peut cependant être prise en charge ; les patients qui ont une activité sexuelle préopératoire répondent au traitement par injection intracaverneuse. D'autres traitements sont en cours d'évaluation dans ce contexte.

La décision du traitement dépend donc pour les stades localisés essentiellement des caractéristiques de la lésion et de l'espérance de vie du patients. Une fois le patient informé des risques et de l'intérêt de chacun des traitement, la conduite à tenir la plus appropriée au patient et à la maladie et à son espérance de vie sera définie.

\section{INDICATIONS DU TRAITEMENT HORMONAL ET PRINCIPES}

La déprivation androgénique (castration) est justifiée dans le cas du cancer prostatique métastasé et peut être discutée dans le cas d'un traitement néo-adjuvant de la radiothéra- 
pie pour les stades T3 (Bolla et al., 1997), le traitement néo-adjuvant de la chirurgie dans les stades T2, n'ayant pas montré son efficacité [1].

Longtemps la castration chirurgicale par orchidectomie bilatérale est restée le traitement de référence. Il s'agit d'une intervention très simple, bien tolérée, qui est responsable de la diminution de $95 \%$ de la testostérone circulante à un niveau de 10 à $50 \mathrm{ng} / 100 \mathrm{ml}$ [11].

Plus récente, l'utilisation des agonistes de la LHRH représente un progrès certain. Les agonistes de la synthèse de la LHRH, ont une demi-vie longue et sont aussi efficaces que les oestrogènes et la castration chirurgicale (The Leuprolide Study Group, 1984), (Veterans Administration Cooperative Urological Research Group, 1967). Du fait de leur excellente tolérance, ils sont le plus souvent utilisés surtout lorsque le choix est donné au patient. Il existe maintenant des formes injectables tous les trois mois.
Globalement, le taux de réponse à la déprivation androgénique est de 80 à $90 \%$ des patients ayant une tumeur prostatique avec métastases. Dix à $20 \%$ d'entre eux sont d'emblée résistants au traitement et ont un mauvais pronostic immédiat. Il s'agit d'un traitement non curatif et, malgré la disparition parfois spectaculaire des métastases et l'amélioration de l'état général, la durée de réponse est en moyenne de 18 à 36 mois selon les groupes pronostiques [9]. A ce stade d'échappement au traitement hormonal, la durée moyenne de survie est d'environ 10 mois [19, 21] (Figure 2).

Le dosage du PSA seul n'est pas un critère de réponse. Bien que sa baisse significative au dessous des $10 \mathrm{ng} / \mathrm{ml}$ au troisième mois soit un facteur de bon pronostic [29], la définition de réponse complète ou partielle fait surtout intervenir des critères cliniques, biologiques et radiologiques (tableau 2).

La surveillance des patients traités par la déprivation androgénique est recommandée au

\section{Survie actuarielle au stade des métastases}

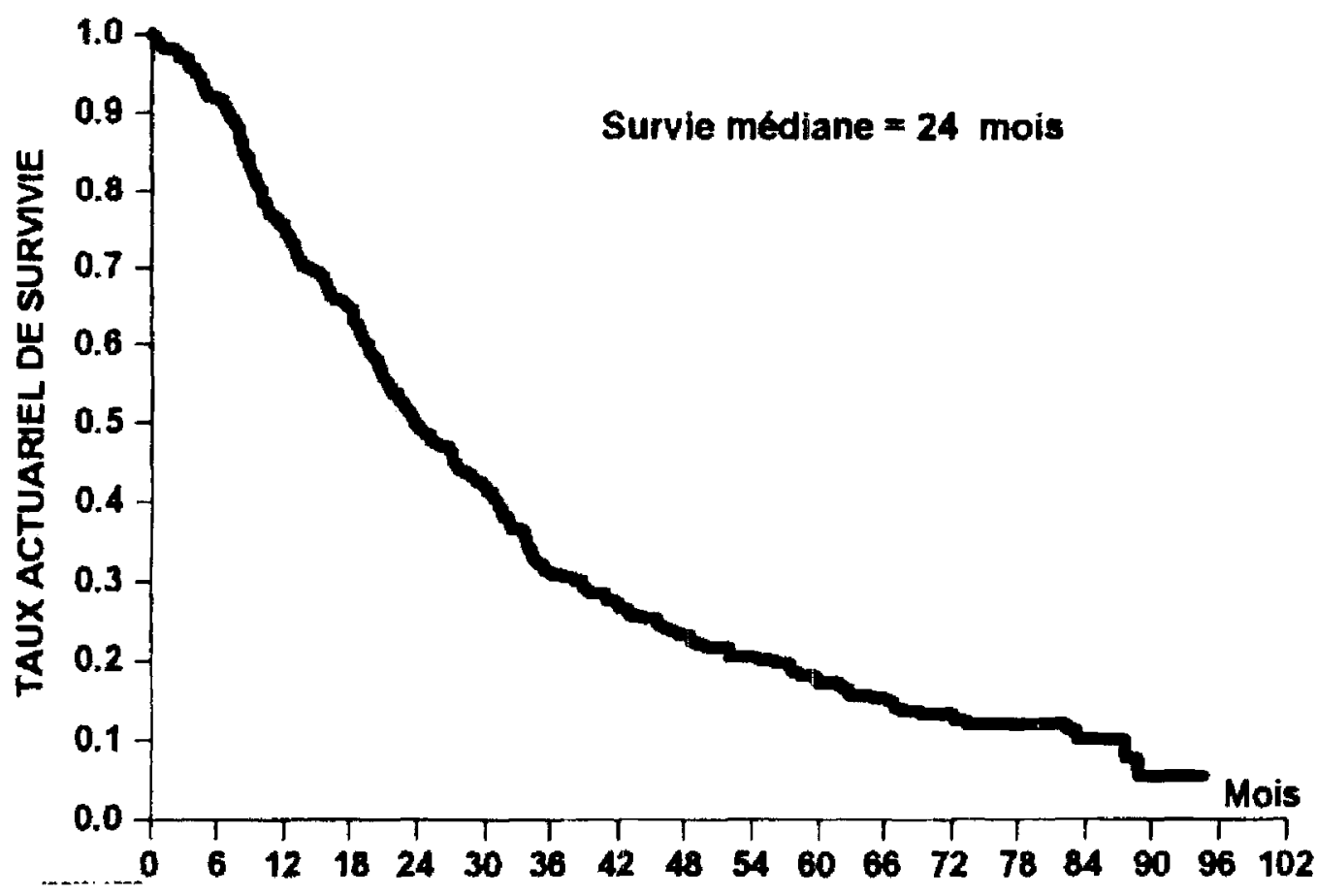

Figure 2 : courbe de survie spécifique actuarielle d'une cohorte de patients T2-T4, M+ traités par blocage androgénique complet 
moins tous les 6 mois [20] et doit comprendre outre le dosage systématique du PSA, un examen clinique complet avec un toucher rectal et la surveillance de la fonction rénale. Tous les signes de progression non spécifiques orientent les examens complémentaires qui ne doivent pas pour autant être prescrits de manière systématique. Il semble que $50 \%$ des patients ont une progression objective sur des critères spécifiques ou non spécifiques sans avoir d'élévation des PSA (Colombel, communication, AFU 1997).

Le renforcement de la déprivation androgénique par l'utilisation des anti-androgènes a été proposé pour pallier l'insuffisance de réponse obtenue par castration seule. Les résultats initiaux ont été très prometteurs [18]. Toutefois, les résultats des études randomisées sont contradictoires, de même les métaanalyses [3], (Prostate Cancer Trialist's Collaborative Group, 1995). Ces résultats sont à l'origine d'une certaine confusion en ce qui concerne les indications du blocage androgénique complet.

Le blocage androgénique complet semble présenter un avantage dans les formes de bon pronostic et surtout lorsque la réponse à 3 mois est complète selon les critères décrits [12]. Des études cliniques sont en cours pour déterminer quels sont les groupes pronostiques qui bénéficient effectivement de ce traitement. A l'inverse, lorsque la réponse est incomplète ou lorsqu'il existe une progression biologique ou clinique, il est recommandé de stopper la prescription de l'anti-androgène du fait de la possibilité d'une action agoniste paradoxale sur le récepteur des androgènes.

Au stade des métastases, l'utilisation des antiandrogènes seuls reste du domaine de l'investigation clinique. Les études de comparaison qui ont été réalisées tendent à montrer qu'ils pourraient être utilisés dans certains cas (faible masse tumorale, état général satisfaisant, $\mathrm{PSA}<100 \mathrm{ng} / \mathrm{ml})[4,8,31]$.

Avant traitement, les formes métastatiques ou localement avancées sont parfois responsables de symptômes liés aux complications locales de la progression tumorale. Les complications urologiques (hydronéphrose par obstruction urétérale ou adénopathies pelviennes et obstruction vésicale par la tumeur primitive) répondent, dans $70 \%$ des cas et dans un délai de 4 à 12 semaines, à la déprivation androgénique. Il est parfois nécessaire de dériver les urines par néphrostomie percutanée ou la mise en place de sondes urétérales, lorsqu'il existe une souffrance rénale obstructive. En cas de rétention vésicale, le raisonnement est le même, il faut dériver les urines vésicales par la mise en place d'un catheter ou d'une sonde vésicale en fonction du degré de la rétention. La résection endoscopique d'un cancer de prostate est un geste difficile à haut risque d'incontinence postopératoire et sera donc réservée aux formes résistantes au traitement hormonal ou lors de l'échappement, d'où l'importance de disposer d'un bilan préopératoire complet (PSA, TR) chez un patient de moins de 75 ans et en rétention. La radiothérapie est parfois envisagée, elle est alors synergique de l'effet de la déprivation hormonale [10].

Le traitement des complications osseuses liées aux métastases repose sur la déprivation androgénique qui est efficace dans $80 \%$ des cas sur les symptômes. Le traitement peut être associé à une corticothérapie. Il faut redouter à ce stade les complications nerveuses par compression des métastases osseuses, particulièrement au niveau du rachis. Le diagnostic de compression médulaire ou d'épidurite doit être précoce afin de permettre une prise en charge (chirurgicale ou par radiothérapie) le plus tôt possible. Dans ce cas, la déprivation androgénique doit être immédiate, associant corticothérapie à forte dose (Solumedrol $1000 \mathrm{mg} / 24 \mathrm{~h}$ ) à une castration chirurgicale. Malgré le pronostic sombre à ce stade de la maladie, surtout en cas d'échappement hormonal, il est parfois nécessaire de réaliser une laminectomie postérieure associée à une radiothérapie ou bien une radiothérapie seule. Il peut également être nécessaire de prévenir les complications osseuses à type de fractures par la radiothérapie.

Les complications parenchymateuses et hématologiques répondent elles aussi rapidement à la castration chirurgicale et la corticothérapie à forte dose.

Ces principes s'appliquent également au stade de l'échappement hormonal où le traitement 
symptomatique est le plus important, le principal objectif étant de garantir une qualité de vie convenable. Il est clair que la prévention des complications, une alimentation assistée et le traitement des douleurs permettent de limiter les journées d'hospitalisation en fin de vie.

\section{LES THÈMES DE RECHERCHE ACTUELS}

Les progrès les plus importants de ces dernières années dans le domaine du cancer de la prostate l'ont été dans le domaine de l'anatomie chirurgicale et de la technique de la chirurgie du petit bassin chez l'homme. Il s'agit d'un domaine perfectible, en particulier dans la prévention de l'impuissance postopératoire. Les recherches de substitution nerveuse per opératoire, les nouveaux traitements post-opératoires et la prise en charge de l'impuissance lorsqu'elle représente un handicap sont des voies de recherche très actives. Le contrôle carcinologique des formes localement avancées, la prédiction préopératoire du stade pathologique représentent également de thèmes de recherche en développement constant. Nous attendons de l'avenir de nouveaux marqueurs de l'agressivité tumorale comme l'angiogénèse tumorale, ou des marqueurs du franchissement capsulaire comme la présence de cellules circulantes dans le sang périphérique que nous détectons par PCR. Il faut compter sur l'évaluation de nombreux marqueurs à partir de fragments de biopsies de très faible volume, et pour cela nous attendons beaucoup des nouvelles technologies issues des empreintes d'ADN.

Au stade des métastases et du traitement hormonal, les recherches sont actuellement orientées vers une meilleure compréhension des phénomènes de mort cellulaire (apoptose) des cellules prostatiques qui apparaissent au décours de la castration. Il s'agit d'un processus qui dépend de l'expression de gènes. Comme tout phénomène biologique, l'apoptose est sous le contrôle de gènes activateurs et inhibiteurs qui vont donc moduler l'action de la castration dans l'exemple du cancer de la prostate et donc moduler la réponse au traitement. Ces mécanismes sont donc importants à comprendre avant de développer de nouveaux médicaments ou formes de traitements. Dans le cas de la résistance au traitement et de l'échappement hormonal, la thérapie génique est une issue possible qui aurait pour objectif de rendre réversible la résistance au traitement.

$\mathrm{Du}$ demi siècle qui nous sépare de la publication de Huggins sur l'effet de la castration dans le cancer prostatique, on pourrait dire que de nombreux progrès ont été réalisés qui permettent aux formes localisées d'être guéries. Pour les formes localement avancées et au stade des métastases, malgré l'utilisation de nouvelles formes de traitements hormonaux, aucun progrès thérapeutique n'a été réalisé, la réponse au traitements est limitée et l'échappement hormonal est inéluctable. Les progrès de la recherche fondamentale, appliquée au cancer de prostate devraient nous permettre d'améliorer la prise en charge de ces patients.

\section{RÉFÉRENCES}

1. ABBAS F. \& SCARDINO P. Why neoadjuvant androgen prior to radical prostatectomy unnecessary. Urol.Clin.North Am. 1996, 23, 587-614.

2. AMSELLEM D., OGIEZ N., SALOMON L., CHOPIN D., ABBOU C.C. \& COLOMBEL M. Un PSA <à $20 \mathrm{ng} / \mathrm{ml}$ exclu t-il le diagnostic de cancer de prostate métastatique. Prog.Urol. 1998 (In Press)

3. BERTAGNA C., DEGERY A., HUCHER M., FRANCOIS J.P. \& ZANITARO J. Efficacy of the combination of nilutamide plus orchiectomy in patients with metastatic prostatic cancer. A meta-analysis of seven radnomized double blind trials (1056 patients). Brit.J.Urol. 1994, 73, 396-402.

4. BOCCON GIBOD L., FOURNIER G., BOTTET P., MARECHAL J.M., GUITER J., RISHMAN P., HUBERT J., SORET J.Y., MANGIN P., MALLO C. \& ESCTEIN-FRAYSSE E. Flutamide versus orchiectomy in the treatment of metastatic prostate carcinoma. Europ.J.Urol. 1997, 32, 391-396.

5. BOLLA M., GONZALLES D., WARDE P., DUBOIS J.B., MIRMANOFF R., STORME G., BERNIER J., KUTEN A., STERNBERG C., GIL T., COLLETTE L. \& PIERART M. Improved survival in patients with locally advanced prostate cancer treated with radiotherapy and goserelin. N.E.M.J. 1997, 337, 295-245.

6. CATALONA W.J., RICHIE J.P., AHMANN F.R., HUDSON M.A., SCARDINO P.T., FLANIGAN R.C., DEKERNION J., RATLIFF T.L., KAVOUSSI L.R., DALKIN B.L., WATERS B., MACFARLANE M.T. \& SOUTHWICK P.C. Comparison of digital rectal examination and serum prostate specific antigen in the 
early detection of prostate cancer: results of multicenter clinical trial of 6630 men. J.Urol. 1994, 151, 1283-1290.

7. CATALONA W.J., SMITH D.S., RATLIFF T.L. \& BASLER J.W. Detection of organ confined prostate cancer is increased through prostate specific antigen based screening. JAMA 1993, 270, 104

8. CHODAK G., SHARIFI R., KASIMIS B., BLOCK N., MACRAMALLA E. \& KENNEALEY G.T. Single agent therapy with bicatulamide: a comparison with medical or surgical castration in the treatment of advanced prostate carcinoma. Urology 1995, 46, 855

9. CHODAK G.W., VOGELZANG N.J., CAPLAN R.J., SOLOWAY M.S. \& SMITH J.A. Independent prognostic factors in patients with metastatic (stage D2) prostate cancer. JAMA 1991, 265, 618-621.

10. COLOMBEL M., MALLAME W. \& ABBOU C.C. Influence of urological complications on the prognosis of prostate cancer. Eur.Urol. 1997, 31 Suppl , 3, 2124.

11.DANESHGARI F. \& CRAWFORD E.D. Endocrine therapy of advanced carcinoma of the prostate. Cancer 1993,71, 1089-1097.

12. DIJKMAN G.A., JANKNEGT R.A., DE REIJKE T.M. \& DEBRUYNE F.M.J. Long term efficacy and safety of nilutamide plus castration in advanced prostate cancer and the significance of early prostate specific antigen normalization. J.Urol. 1997, 158, 160-163.

13. FOWLER J.E., TERREL F.L. \& RENFROE D.L. Comorbidity and survival of men with localized prostate cancer treated with surgery or radiation therapy. J.Urol 1996, 156, 1714-1718.

14.GLEAVE M.E., COUPLAND M., DRACHENBERG D., COHEN L., KWONG S., GOLDENBERG S.L. \& SULLIVAN L.D. Ability of serum prostate specific antigen levels to predict normal bone scans in patients with newly diagnosed prostate cancer. Urology 1996,47 , 708-712.

15. HANKS G.E., HANLON A.L., SCHULTHEISS T.E., FREEDMAN G.M., HUNT M., PINOVER W.H. \& MOVSAS B. Conformal external beam treatment of prostate cancer. Urology (1997, 50, 87-92.

16. JOHNSTONE P.A., POWELL C.R., RIFFENBURGH R., ROHDE D.C. \& KANE C.J. Second primary malignancies in T1-3N0 prostate cancer patients treated with radiation therapy with 10-year followup. J.Urol 1998, 159, 946-949.

17.KUBAN D.A., EL-MAHDI A.M. \& SCHELLHAMMER P.F. Prognosis in patiens with local recurrence after definitive irradiation for prostatic carcinoma. Cancer 1989, 63, 2421-2425.

18. LABRIE F., DUPONT A. \& BELANGER A. New hormonal therapy in prostatic carcinoma: combined treatment with LHRH agonist with an antiandrogen. Clin.Invest.Med. $1982,5,267-275$.

19. MATZKIN H., SOLOWAY M.S., SCHELLHAMMER P.F., CHODAK G., SMITH J.A., CAPLAN R. \& KENNEALEY G.T. Prognostic factors in stage D2 prostate cancer treated with pure nonsteroidal antiandrogen.
Cancer 1993, 72, 1286-1290.

20. MURPHY W.M., SLACK N.H. \& PARTICIPANTS IN THE NATIONAL PROSTATIC CANCER PROJECT Current status of the National Prostatic Cancer Treatment Protocols. In Controlled Trials in Urologic Oncology. Eds L. Denis, W.M. Murphy, G.R. Prout \& F.H. Schroeder. New York: Raven Press. 1997, pp. 119133.

21. NEWLING D. The palliative therapy of advanced prostate cancer with particular reference to the results of recent European clinical trials. Brit.J.Urol. 1997, 79, 72-81.

22. OEFELEIN M.G., SMITH N.D., GRAYHACK J.T., SCHAEFFER A.J. \& MCVARY K.T. Long term results of radical retropubic prostatectomy in men with high grade carcinoma of the prostate. J.Urol. 1997, 158, 1460-1465.

23. PANNEK J., RITTENHOUSE H.G., CHAN D.W., EPSTEIN J.I., WALSH P.C. \& PARTIN A.W. The use of percent free prostate specific antigen for staging clinically localized prostate cancer. J.Urol. 1998, 159, 1237-1242.

24. PARKER S.L., TONG T., BOLDEN S. \& WINGO R.A. Cancer statistics. 1997, CA 47, 5

25. PARTIN A.W., KATTAN M.W., SUBONG E.N.P., WALSH P.C., WOJNO K.J., OESTERLING J.E., SCARDINO P. \& PEARSON J.D. Combination of prostatic specific antigen, clinical stage, and Gleason score to predict pathological stage of localized prostate cancer. JAMA 1997, 277, 1445-1451.

26. POWELL C.R., HUISMAN T.K., RIFFENBURGH R. H., SAUNDERS E.L., BETHEL K.J. \& JOHNSTONE P.A.S. Outcome for surgically stagged localized prostate cancer treated with external beam radiation therapy. J.Urol. 1997, 157, 1754-1759.

27. PROSTATE CANCER TRIALIST'S COLLABORATIVE GROUP Maximum androgen blockade in advanced prostate cancer: an overview of 22 randomised trials with 3283 deaths in 5710 patients. Lancet 1995, 346, 265-269.

28. SCARDINO P., WEAVER R. \& HUDSON M.A. Early detection of prostate cancer. Hum.Path 1992, 23, 211

29. SMITH J.A., LANGE P.H., JANKNEGT R.A., ABBOU C.C. \& DEGERY A. Serum markers as a predictor of response duration and patient survival after hormone therapy for metastatic carcinoma of the prostate. J.Urol. 1997,157, 1329-1334.

30. SOH S., KATTAN M.W., BERKMAN S., WHEELER T.W. \& SCARDINO P. Has there been a recent shift in the pathological features and prognosis of patients treated with radical prostatectomy. J.Urol. 1997, 157, 2212-2218.

31. SOLOWAY M.S., SCHELLHAMMER P.F., SMITH J.A., CHODAK G.W. \& KENNEALEY G.T. Bicatulamide in the treatment of advanced prostatic carcinoma: a phase II multicenter trial. Urology 1996, 47, 33-37.

32. STAMEY T.A. Making the most out of six systematic sextant biopsies. Urology 1995, 45, 2-12. 
33. THE LEUPROLIDE STUDY GROUP Leuprolide versus diethylstilbestrol for metastatic prostate cancer. N.E.M.J. 1984, 311, 1281-1286.

34.ETERANS ADMINISTRATION COOPERATIVE UROLOGICAL RESEARCH GROUP. Treatment and survival of patients with cancer of the prostate. Surg.Gynecol.Obstet. 1967,124, 1011-1017.

35. WALSH P.C. \& DONKER P.J. Impotence following radical prostatectomy: insight into etiology and prevention. J.Urol. 1982, 128, 492

\begin{abstract}
Prostate Cancer : Acquired knowledge and challanged for year 2000
\end{abstract}

M. Colombel, F. Blanc, J.M. Marechal

Principles of the diagnosis and treatment of prostate cancer at any stage are still improving. Early diagnosis is accessible throughout the use of the PSA test associated with digital rectal examination which lead to indicate transrectal biopsies. This allowed to treat patients at an earlier stage and significantly improved prognosis in the case of organ confined disease. Progress made in the radical prostatectomy technique have contributed to decrease the postoperative morbidity and is the treatment of reference in clinically localized disease. Radiation therapy still remains a valuable alternative, however, results are more difficult to evaluate. Hormonal treatment using androgen deprivation is indicated at the stage of metastasis. LHRH agonist associated with anti antiandrogens are as much efficacious as surgical castration. Unfortunately, the prognosis of advanced disease remains unpredictable. Objectives for the future will be to improve the diagnostic and staging of prostate cancer et to better define therapeutic indications; better understand the effects of androgen deprivation; and to propose new therapies for hormone refractory cancers.

Key-words: Prostate cancer; diagnosis; treatment; pathophysiology 